needed; these are often laborious and not practical for high-throughput screening. In order to develop a rapid automated mutation detection method, we have evaluated denaturing high-performance liquid chromatography for detection of TP53 mutations in tumor tissue. The specificity and sensitivity of the method were compared to those of denaturing gradient gel electrophoresis through testing on standard-quality DNA preparations as well as on DNA extracted from formalin-fixed, paraffin-embedded tissue sections.

Amler, Lukas

\section{Functional dissection of transcriptional profiles in androgen-dependent and - independent prostate cancer}

Lukas Amler ${ }^{1}$, David Agus ${ }^{2}$, Nicholas Dracopoli ${ }^{1}$, Howard Scher ${ }^{2} \&$ Garret Hampton ${ }^{3}$

${ }^{1}$ Bristol-Myers Squibb, Princeton, New Jersey, USA

${ }^{2}$ Memorial Sloan-Kettering Cancer Center, New York, New York, USA

${ }^{3}$ Genomics Institute of the Novartis Research Foundation, La Jolla,

California, USA

Using microarray analysis, we have measured changes in gene expression that occurred during the evolution of a primary prostate cancer xenograft, CWR22, from normalcy to relapsed, androgen-independent growth following androgen deprivation therapy. Rather than relying on simple pairwise comparison of the data between successive biological states (for example, normal versus tumor), we clustered the expression data on the basis of behavior in multiple biological states, representative of the entire process of prostate cancer progression. By examining the data in the context of different proliferative conditions, before and after hormonal manipulation in the xenograft model, we could identify groups of genes associated with proliferation, metabolism, hormone responsiveness and malignant transformation, as well as a small number of genes directly associated with androgen-independent growth. Our results imply that the evolution to androgen independence is due, in part, to reactivation of the androgen-response pathway in the absence of androgens, but that this reactivation is probably incomplete.

Amler, Lukas

\section{Dysregulated expression of androgen- responsive and nonresponsive genes in the androgen-independent prostate cancer xenograft model, CWR22-R}

Lukas Amler $^{1}$, David Agus ${ }^{2}$, Carrie LeDuc ${ }^{1}$, Lisa Sapinoso ${ }^{1}$, William Fox ${ }^{2}$, Suzanne Kern ${ }^{1}$, Dori Lee ${ }^{1}$, Vivian Wang ${ }^{1}$,

Mauri Leysens ${ }^{1}$, Brian Higgins ${ }^{2}$, Jason Martin ${ }^{1}$,

William Herald ${ }^{3}$, Nicholas Dracopoli ${ }^{1}$, Carlos Cordon-Cardo ${ }^{3}$, Howard Scher ${ }^{2} \&$ Garret Hampton ${ }^{1}$

${ }^{1}$ GENOS Biosciences, Inc., La Jolla, California, USA

${ }^{2}$ Department of Medicine, Memorial Sloan-Kettering Cancer Center, New York, New York, USA

${ }^{3}$ Department of Pathology, Memorial Sloan-Kettering Cancer Center, New York, New York, USA

Treatment of metastatic prostate cancer with androgen ablation often elicits dramatic tumor regressions, but the response is rarely complete, making clinical recurrence inevitable with time. To gain insight into therapy-related progression, we used microarray analysis to monitor changes in gene expression that occurred following androgen starvation of an androgen-dependent prostate tumor xenograft, CWR22, and the emergence of an androgen-independent tumor, CWR22-R. Androgen deprivation arrested the growth of CWR22 cells, as evidenced by decreased expression of genes encoding cell cycle components and basal cell metabolism, respiration and transcription, as well as the induced expression of putative negative regulatory genes that may act to sustain cells in a nonproliferative state. Evolution of androgen-independent growth and proliferation, represented by CWR22-R, was associated with a re-entry into the active cell cycle and the upregulation of several genes that were expressed at low levels or absent in the androgen-dependent tumor. Restoration of androgen to mice bearing androgenindependent CWR22-R tumors induced, augmented or repressed the expression of several androgen-responsive genes. Two of the genes whose expression was increased by androgens (the calcium-binding protein, S100P, and the FK-506binding protein, FKBP51) were also expressed in CWR22-R. The dysregulated behavior of these genes is analogous to that of other androgen-dependent genes (for example, prostate-specific antigen and human kallikrein 2), which are commonly re-expressed in androgen-independent disease in the absence of androgens. These results imply that evolution to androgen independence is due in part to reactivation of the androgen response pathway in the absence of androgens, but that this reactivation is probably incomplete.

Aprelikova, Olga

[10]

\section{Differential gene expression in cells with targeted deletion of BRCA1 tumor suppressor}

\author{
Olga Aprelikova ${ }^{1}$, Amy Pace ${ }^{2}$, Beverly Koller $^{2} \&$ Edison Liu ${ }^{1}$ \\ ${ }^{1}$ Section of Molecular Signaling and Oncogenesis, Division of Clinical Sciences, \\ National Cancer Institute, Bethesda, Maryland 20892, USA \\ ${ }^{2}$ Lineberger Comprehensive Cancer Center, University of North Carolina at \\ Chapel Hill, Chapel Hill, North Carolina 27599, USA
}

$B R C A 1$ plays a role in DNA repair and transcription, although the importance of the transcriptional control to the clinical phenotype is unclear. To identify transcriptional targets of $B R C A 1$, we assessed differential gene expression in mouse embryonic stem cells with deletion of both BRCA1 alleles using high-density microarrays. Several groups of genes were downregulated in the knockout cell line, including some stress response genes, cytoskeleton genes and genes involved in protein synthesis and degradation. Of particular interest was the finding that the 14-3-3 $\sigma$ checkpoint control gene was significantly downregulated in $B R C A 1^{-1-}$ cells when compared with wild-type embryonic stem cells or $B R C A 1^{-1-}$ embryonic stem cells reconstituted with a $B R C A 1$ transgene. Irradiation of $B R C A 1^{-1-}$ cells showed much lower levels of 14-3-3 $\sigma$ induction. Moreover, when these cells were treated with ionizing radiation their cell cycle profile showed an inability to sustain the G2/M growth arrest typical of cells deprived of 14-3-3 $\sigma$. We found that $B R C A 1$ synergistically activates p53-dependent transcription of the 14-3-3 $\sigma$ gene and that this activation is dependent on an intact $\mathrm{p} 53, B R C A 1$ and $\mathrm{p} 53$ response elements. These data indicate that BRCA1 is an important transcriptional cofactor in p53-mediated responses to DNA damage and may explain some of the clinical and laboratory presentations of cancers in $B R C A 1$ carriers. 